\title{
Amphitryon d'Ignacio Padilla : échecs et crise de la filiation
}

\section{Emilie Etemad-Kasaeyan}

\section{(2) OpenEdition}

1 Journals

Édition électronique

URL : https://journals.openedition.org/cher/12269

DOI : $10.4000 /$ cher.12269

ISSN : 2803-5992

Éditeur

Presses universitaires de Strasbourg

\section{Édition imprimée}

Date de publication : 30 juin 2012

Pagination : 145-154

ISBN : 978-2-35410-046-9

ISSN : 1968-035X

\section{Référence électronique}

Emilie Etemad-Kasaeyan, «Amphitryon d'Ignacio Padilla : échecs et crise de la filiation », reCHERches [En ligne], 8 | 2012, mis en ligne le 21 février 2022, consulté le 24 février 2022. URL : http:// journals.openedition.org/cher/12269; DOI : https://doi.org/10.4000/cher.12269

\section{(c) (i) (ㅇ)}

Ce(tte) œuvre est mise à disposition selon les termes de la Licence Creative Commons Attribution Pas d'Utilisation Commerciale - Partage dans les Mêmes Conditions 4.0 International. 


\title{
Amphitryon d'Ignacio Padilla: échecs et crise de la filiation
}

\author{
Emilie Etemad-KasAeyan \\ Université Rennes 2
}

$\mathrm{D}$ ans l'introduction de l'ouvrage collectif qu'il dirige en 1998 intitulé Echiquiers d'encre. Le Jeu d'échecs et les Lettres (XIX ${ }^{e}-X X^{e}$ siècles), Jacques Berchtold écrit:

Perçue dans la perspective restreinte de la psychanalyse - et quelle que puisse être l'attitude rationnelle consciente adoptée par le joueur -, la poursuite de la mise «échec et mat» (la paralysie du roi adverse) est un acte équivalent symboliquement au meurtre ou à la castration du père. Cette attitude hostile se projette sans danger sur l'échiquier et réalise son transfert dans l'ordre symbolique, sublimant ainsi l'agressivité du processus œedipien, jusqu'à la destruction du roi dans le jeu du «père» rival (Berchtold 1998: 17-19).

Cette confrontation symbolique au père relevée par la psychanalyse dans le jeu d'échecs est reprise sous forme de motif structurant dans Amphitryon d'Ignacio Padilla. Le roman, paru en 2000, tisse à travers les parties évoquées des relations problématiques au père biologique et symbolique. L'histoire déroule sur trois générations, du début $\mathrm{du} \mathrm{xx}^{\mathrm{e}}$ siècle à Vienne, à Londres en 1989, un réseau d'échanges d'identités et de manipulations dont le nœud est Thadeus Dreyer. Ce nom passe d'un jeune soldat de la Première Guerre Mondiale à un Juif fuyant son passé (Jacob Efrussi) puis se transmet comme une malédiction à un homme en quête de reconnaissance (Richard Schley). L'échange d'identité est à chaque fois l'enjeu d'une partie d'échecs.

Or le premier Thadeus Dreyer devenu Viktor Kretzschmar élève sans le savoir le fils illégitime du troisième Dreyer, l'homme qu'il souhaite voir périr. Qu'il s'agisse de fils découvrant leur bâtardise (Franz T. Kretzschmar) 
ou de créatures cherchant à arracher leur âme des mains de leur créateur (Viktor Kretzschmar, Jacob Efrussi), la relation à l'ascendance est toujours conflictuelle. L'enjeu pour les personnages masculins du roman n'est rien de moins que se protéger contre la menace constante d'une perte identitaire: n'étant fils de personne ou créatures de "pères» qui les dévorent, ils ne parviennent pas à s'ancrer dans leur être.

Cette recherche identitaire se traduit par deux pulsions: le parricide, qui n'est jamais mené à terme - que ce soit sur un mode réel ou symbolique et le suicide. Les fils ne parviennent pas à triompher de leurs pères: Franz, en perdant la partie d'échecs, devient la créature de son géniteur, Richard Schley alias Thadeus Dreyer. De même, Jacob Efrussi ne parvient pas à se libérer de l'emprise paternelle - celle-là même qui le force à se mesurer à tous les adversaires de Vienne dans des parties dont l'enjeu n'est pas de gagner aux échecs mais de souligner la suprématie juive - si ce n'est en renonçant à son nom et à son identité, en se condamnant à devenir une âme errante et faustienne.

L'unique solution tenable pour reprendre son identité et se saisir de son destin consiste donc dans l'acceptation de la mort, seule capable d'arracher la créature à son créateur: or le cadre historique du roman - l'Europe de la Première et Deuxième Guerre Mondiale - rend pleinement effective cette pulsion. S'il est donc question d'échec dans le roman c'est bien de celui de la filiation: aucune transmission n'aboutit et les différents échanges identitaires (l'enjeu même des parties d'échecs) ne mènent qu'à des impasses.

Cette lecture "psychologisante» rejoint une analyse nettement plus littéraire: il me semble en effet que le "jeu» sur la relation aux pères biologiques dans le roman couvre une réflexion plus sourde sur la filiation aux grands auteurs. Partant du désir de lire le roman en regard du Manifeste Crack de 1996, il m’apparaît qu'Ignacio Padilla assume la nécessité de la continuité à travers un dialogue constant avec la littérature qui le précède. On peut bien entendu penser d'abord à toute la littérature des échecs (Lewis Carroll, Nabokov, Faulkner, Zweig pour n'en citer que quelques-uns) et en particulier au poème «Ajedrez" de Borges ${ }^{1}$.

Mais ce qui m'apparaît plus nettement, c'est la relation particulière à un intertexte précis: le sonnet de Pessoa, présent sous forme de citation en épigraphe du roman. Or Pessoa rejoint en tous points la problématique

1 Si l'on suit l'analyse proposée par Nina Pluta dans Tendencias de la narrativa mexicana actual. 
identitaire évoquée précédemment et offre sans doute une réponse littéraire à l'échec de la filiation biologique: devant la menace d'une dissolution identitaire, face au péril d'une dépersonnalisation engagée dans l'époque des deux Guerres Mondiales, une solution est proposée: sortir de la dépersonnalisation (l'échec de la personne) pour entrer dans une pluripersonnalité assumée (la personne littéraire). Or cette promotion du multiple rejoint à mon sens les grands principes évoqués dans le Manifeste Crack: refus d'une littérature nationale ou engagée, recherche formelle plurielle, ouverture aux influences mondiales.

Je propose donc une lecture du roman qui passerait de l'échec de la filiation biologique aboutissant à la dépersonnalisation, à une porosité maximale de la personne littéraire comme conséquence de l'acceptation de la filiation lettrée.

\section{Pères infanticides et menace de dépersonnalisation}

Comme évoqué en introduction, la menace de la dépersonnalisation constitue un thème majeur d'Amphitryon. L'angoisse liée à la perte de l'identité se décline sur plusieurs plans.

Un plan familial et biologique d'abord, puisque la plupart des pères du roman "dévorent» l'âme de leurs fils. Richard Schley comme Jacob Efrussi sont les instruments d'une rivalité de pères dans la Vienne du début du siècle: à travers les parties d'échecs menées, ce sont deux systèmes de «valeurs» qui s'opposent:

Casi pude escucharle cuando arrastraba los pies camino de la sinagoga, como si la sola idea de ostentar innecesariamente su condición de judío por las calles de Viena le impusiese un peso insostenible. Invoqué también sus numerosos éxitos de precoz genio del ajedrez, conseguidos siempre bajo la mirada vigilante de su padre, quien se esmeraba por hacer de aquellas victorias pueriles una demostración pública de la superioridad de su gente. Más que un juego, el ajedrez era para el joyero la prueba inequívoca de que una identidad colectiva y genial había sido sembrada en su hijo tras milenios de persecuciones, diásporas y defensas temerarias de una consciencia de raza mantenida con dolor y sangre (Padilla: 75-76).

L'enjeu pour Jacob Efrussi et Richard Schley consiste donc à se dégager de la mainmise paternelle et à s'affirmer dans leur être propre. Or la solution d'Efrussi, échanger son identité avec de multiples autres via les parties d'échecs, n'aboutit qu'à la perte de son être profond. Quant à Richard Schley, il prend d'abord l'identité du père Wagram puis assume celle 
d'Efrussi devenu Thadeus Dreyer. Mais en jouant le jeu des substitutions, il accepte aussi de se perdre:

Efrussi no estaba loco, más bien pensaba con la lógica apabullante de los derrotados, con la resignación última de un hombre expuesto a huir continuamente de una identidad que siempre le había parecido demasiado onerosa y precisa como para poder soportarla. De alguna manera, ambos habíamos querido escapar en el pasado a nuestra condición, nuestra raza y la fe de nuestros padres, y ahora, por tanto, debíamos resignarnos y enfrentar la inutilidad de aquella fuga (99-100).

Impossible donc d'échapper à la toute-puissance du père ou à la menace d'une perte identitaire si ce n'est en devenant soi-même le «bourreau» de son fils. Richard Schley se transforme ainsi à son tour en père omnipotent: ayant pris l'identité de Thadeus Dreyer, il condamne son fils illégitime Franz T. Kretzschmar à perdre sa propre identité pour se substituer à Adolf Eichmann. Il remarque lui-même à la fin de sa vie qu'il a «volé » l'âme de son fils. Au directeur de l'hôpital de Francfort où le premier Thadeus Dreyer est enfermé, Richard Schley devenu le Baron Block déclare en parlant de Franz: "Yo di la vida a ese hombre, señor mío, pero le robé el alma. En verdad, hoy daría cualquier cosa para devolvérsela» (208). Malheureusement son fils, s’il s'agit bien du faux Adolf Eichmann (et le récit préserve l'incertitude) sera pendu à Tel-Aviv avant qu'on ait pu révéler l'usurpation.

Plan familial et plan historique se lient donc dans le roman où les fils deviennent des pions des conflits idéologiques mais aussi des combats militaires. La menace n'est en effet pas effective qu'à l'échelle des personnages, elle traverse toute la période qui sert de cadre à la diégèse. D'une guerre à l'autre une même remarque émerge: la guerre renvoie à néant le concept même d'identité individuelle. A plusieurs moments les personnages placés au cour des conflits sont saisis par le constat de dépersonnalisation engendrée dans le conflit armé massif (industriel, moderne). Or cette dépersonnalisation est à la fois vécue dans la douleur et le soulagement - elle enlève le "poids» d'un nom trop lourd à porter tout en faisant basculer la personne dans un anonymat mortifère. Le roman revient à plusieurs reprises sur cette idée:

En esa guerra que parecía prolongarse hasta el infinito, tarde o temprano todos los hombres terminarían desangrándose en la misma trinchera. Y sus nombres, como sus vidas, se igualarían al fin en el más rotundo de los anonimatos (22-23).

lo más sensato era renunciar no sólo al propio nombre, sino a todo aquello que conforma la identidad de los seres condenados a morir (65). 
Dans le contexte militaire moderne, l'anonymat prédomine. Cette «massification» visible dans la mort commune - le massacre collectif - est aussi une composante de la vie politique du $\mathrm{XX}^{\mathrm{e}}$ siècle. Le roman revient sur la dynamique du système totalitaire et son endoctrinement idéologique. En faisant la promotion de l'élan collectif, le régime nazi annihile les consciences individuelles, les volontés propres et se construit sur la dissolution même d'une identité autonome. Franz résume ainsi l'essentiel de l'endoctrinement nazi :

Incluso la memoria de los individuos concretos terminaba por fundirse en el bloque inmenso de un futuro común y grandilocuente donde hombres como mi padre ya no tendrían que preocuparse por sus mezquindades, menos aún por la legitimidad de un nombre que se disolvería en el entusiasmo de multitudes anónimas y felices (43).

La dépersonnalisation devient ainsi l'un des traits qui caractérise la période moderne; les identités sont interchangeables et sans contenu, l'individu est dépossédé de sa force propre comme de son propre destin. Comme le dit Jacob Efrussi: «Mi nombre es Legión, porque somos muchos (98). Il n'est pas étonnant alors que dans le champ artistique ce soit le "copiste» qui devienne l'archétype de la modernité. L'artiste n'est plus créateur, il devient la coquille creuse qui fait pauvrement écho à d'autres œuvres qu'il s'approprie en faussaire. Trois «artistes» sont ainsi présents dans la dernière section du roman: une doublure de cinéma, un faussaire en peinture et un nègre - tous présentés comme de piètres figures:

Comenzó por afirmar que no hacía falta ser un genio para saber que Remigio Cossini era ostensiblemente un nombre inventado, aunque por otra parte, agregó, un falsificador de obras de arte podía llamarse como le viniese en gana, pues la verdadera fortaleza de aquel oficio se apoyaba en el más cobarde de los anonimatos. De Fraester no quiso aclarar si aquél era o no su nombre artístico, pero reiteró su desprecio hacia los dobles cinematográficos, sobre todo si éstos, como parecía ser el caso del señor Fraester, habían perdido incluso la capacidad física para merecer impostar al más mediocre de los actores secundarios. En cuanto a mí, concluyó nuestro anfitrión con desenfado, era una pena que mis mejores libros circulasen ahora en establecimientos de segunda mano, firmados por caballeros y damas supuestamente ilustres cuya celebridad, a pesar de todo, no había podido opacar la torpeza estilística de su negro literario (165).

Enfin, dans une perspective ontologique et métaphysique, le trouble identitaire renvoie l'homme à un statut de pion manipulé par un joueur invisible et tout-puissant. On reconnaît ici l'inquiétude de Borges dans son poème «Ajedrez» lorsqu'il évoque la possibilité que le «jeu» soit «infini». 
En ce sens, la référence à la substitution d'Amphitryon par Zeus rend compte d'une inquiétude à se pressentir le jouet d'un "visage invisible", entendu non plus comme père ou Histoire mais peut-être comme puissance divine.

La menace de dépersonnalisation qui traverse le roman est soulignée fortement par la figure d'un double destructeur. Qu'il s'agisse du réseau de doubles mis en place par Dreyer dans le cadre du projet Amphitryon ou par le couple des jumeaux Goliadkine, la relation est toujours vertigineuse. Impossible en effet de savoir si l'Adolf Eichmann pendu en 1962 est vraiment l'ancien nazi ou s'il s'agit de Viktor T. Kretzschmar. Impossible aussi pour Goliadkine de se débarrasser du double qui le hante: le meurtre de son jumeau réel (Piotra) puis la manipulation de son double symbolique (Dreyer) ne lui apportera aucun soulagement. Ce clin d'œil très appuyé au Double de Dostoïevski conjugue la relation conflictuelle au père à la menace du jumeau.

Le double est bien entendu présent dans la configuration même des parties d'échecs (noirs contre blancs mais avec les mêmes figures) vouées à se rejouer de toute éternité dans un échange constant des identités. Le récit travaille à la fois une structure binaire et ternaire des échanges: les échanges identitaires autour de Dreyer sont ainsi au nombre de trois (comme entendu d'avance dans le nom même du personnage, drei-er). Il est alors curieux de constater que de ce réseau d'échanges une seule figure réchappe: Daniel Sanderson, un écrivain raté. Sanderson en effet échappe à la mort et va jusqu'à sortir de son statut d'homme de l'ombre et récupérer son nom. Mais s'il reprend son nom, il devient aussi le porte-parole d'une communauté inquiétante:

Aunque ahora firmo mis libros con mi proprio nombre, en cierto modo sigo escribiendo lo que otros quieren que escriba, diciéndome que una tarde tendré las agallas para rebelarme y buscar el disparo que Bogart interrumpió en Londres (212).

Jouet de Block, manipulé par Cossini, il est possible qu'à la fin du roman Sanderson joue le jeu d'un groupe occulte poursuivant les enjeux nazis pardelà la chute du régime - ce qui est en tout cas frappant c'est que le seul personnage à retrouver son nom soit aussi un écrivain hanté par le discours des morts. Et en ce sens il me semble que le parcours de Sanderson fait profondément écho au sonnet de Pessoa qui ouvre le roman et qu'à travers lui Amphitryon expose, par-delà une angoisse de dépersonnalisation, une 
réflexion sur l'impersonnel littéraire - entendu comme ouverture maximale de la personne littéraire.

\section{Ruptura y continuidad. La palabra es una y la misma}

Il me semble que Pessoa crée à deux reprises un pont entre le Manifeste de 1996 et le roman de 2000. D'abord par l'épigraphe qui renvoie à un sonnet où le sujet lyrique expose la menace d'une dépersonnalisation. Le sonnet «Súbita mão de algum fantasma oculto» expose la perte d'une identité autonome. Le «je» lyrique se dit en effet hanté par un spectre qui le manipule au point d'en faire son ombre. «Je» n'est «personne» hormis le pion d'une volonté sans visage. L'épigraphe du roman se centre sur le second tercet où l'image se condense dans l'expression «je ne suis personne hormis cette ombre / D'une forme invisible à moi » et c'est le motif de l'ombre qui va être particulièrement travaillé chez Padilla dans des couples «ombre / nom ", «ombre/double». D’autre part, la section du Manifeste rédigée par Padilla en 1996 commence par l'exemple de Pessoa. Il le définit comme un écrivain fertile qui engendre à lui seul, dans une nation stérile, une génération littéraire. Pessoa est un «père» fondateur: «Si Pessoa pudo crear él solo toda una generación en una Lisboa dictatorial y yerma de literatura, fue, ideas aparte, por cansancio». Quelle perspective de lecture peut-on tirer de cette présence de Pessoa dans l'œuvre critique et de fiction d'Ignacio Padilla?

En premier lieu, il me semble que la structure narrative d'Amphitryon est composée comme une réponse sous forme de développement à la citation de départ. C'est à travers un dialogue avec Pessoa qu'Ignacio Padilla ordonne les différentes parties de son roman, comme s'il cherchait à résoudre une combinaison mise en branle dans le sonnet de l'épigraphe:

Sinto que sou ninguém salvo uma sombra

De um vulto que não vejo e que me assombra,

E em nada existo como a treva fria.

Les quatre sections du roman reprennent les termes du sonnet pour les articuler et les développer. La première section "Una sombra sin nombre» dont le narrateur est Franz T. Kretzschmar expose la malédiction de son père Viktor Kretzschmar, le véritable Thadeus Dreyer qui, privé de son nom, est condamné à la folie. Au motif inscrit dans le sonnet d'une dépersonnalisation par l'évidement d'une volonté propre s'ajoute celui de la perte du nom. La seconde section du roman, "De la sombra al nombre» dont le narrateur est Richard Schley, expose comment le nom de Dreyer 
circule du premier Dreyer à Jacob Efrussi et de Jacob Efrussi à Schley. La seule possibilité pour sortir de l'ombre selon Schley c'est que son ancien camarade l'appelle par son nom.

Par la suite Schley accepte de devenir l'ombre de Jacob Efrussi en prenant sur lui sa «malédiction» et le nom de Dreyer. La troisième section du roman «La sombra de un hombre» par Alikoshka Goliadkine travaille le motif du double. Goliadkine assassine son jumeau Piotra mais reste hanté par sa mémoire et la projection de l'identité de son frère sur Dreyer ne parviendra pas à le soulager. Le roman propose ainsi une lecture dostö̈evskienne du sonnet initial. La dernière section «Del nombre a la sombra» par Daniel Sanderson revient sur le statut de la voix qui se dit «ombre». Sanderson «sauve» son nom mais son écriture charrie le discours d'autres ombres.

Une seconde piste de lecture possible pour saisir le sens de l'ombre projetée par Pessoa consiste à interroger le statut des narrateurs dans le roman. Au nombre de quatre plus un (l'Appendice final signé par Padilla), ils peuvent être considérés comme des voix autonomes ou comme des hétéronymes du seul écrivain de la diégèse, Sanderson, voire comme des voix spectrales présentes chez l'auteur. Le jeu est ouvert par la référence au poète portugais et la polyphonie d'Amphitryon devient un écho à la multiplicité des voix engendrées par Pessoa. A la menace de la dépersonnalisation répond le jeu d'une multiplication des perspectives, l'éparpillement d'une conscience mobile qui épouse tour à tour différentes perspectives. La structure narrative s'expose de manière très affichée et aboutit sur un "couronnement» ou «point culminant» (traduction plus proche qu' «Appendice» de l'espagnol «Colofón»), par l'auteur lui-même.

Or dans cette ultime section l'auteur suggère ou impose des propositions de lectures qui signalent malicieusement à l'intuition du lecteur que l'ensemble de la construction n'est peut-être à considérer que comme un miroitement des différentes voix qui traversent le créateur. Il joue en effet sur l'autorité de son nom pour affirmer l'existence de certains personnages du roman et expliquer le suicide de Viktor. Il renvoie au personnage de Sanderson comme s'il s'agissait d'un véritable auteur, jouant alors sur la frontière entre réalité et fiction - reprenant ainsi la construction des voix chez Pessoa, un hétéronyme commentant l'œuvre d'un autre et attestant par là son existence. L'aboutissement du roman consiste donc à instaurer un vertige dans l'esprit du lecteur en démultipliant les voix tout en les affirmant comme réelles. Et parmi ces voix il n'est pas uniquement celles 
engendrées par la propre personne de l'auteur, il est aussi celles véhiculées par la mémoire des œuvres.

Il me semble en effet que la référence à Pessoa rejoint le désir de jouer le jeu de la filiation. Pour Padilla et les auteurs du mouvement Crack, la filiation est entendue comme une source nécessaire au vitalisme de l'écriture. Padilla souligne dans le Manifeste la nécessité d'assumer la continuité, de consentir à la poursuite d'un discours littéraire qui s'inscrive dans la continuité des grands «monstres». Comme il l'écrit dans le Manifeste: «La literatura que reniega de su tradición no puede ni debe crecerse en ella. Ningún monstruo niega sus sombras. Novela o anti-novela, espejo contra espejo, sólo así es posible la ruptura en digna continuidad». Aucun monstre ne nie ses ombres, tous les pères fondateurs englobent leur progéniture et la prétention à l'inédit est une illusion. Le mot essentiel, «sombra», l'ombre est ainsi présent dans le Manifeste et une fois de plus il est redressé par un autre terme «espejo ", le miroir.

Il s'agit de se saisir comme double inversé, à la fois même et autre, relançant le mouvement de l'écriture dans un élan conjoint de rupture et de continuité. "Ruptura y continuidad [...] La palabra es una y la misma; la novela, digan lo que digan, viene de siempre y continúa. Rompiéndola, prevalece. En efecto, si no hay nada nuevo bajo el sol, es porque lo viejo vale para la novedad». Une possibilité pour relancer le mouvement de l'écriture consiste justement à souligner la «nouveauté» de l'ancien en le réactualisant.

A mon sens, se saisir des vers de Pessoa comme ferments pour l'écriture romanesque est une façon de manifester que rupture et continuité sont un même mouvement. C'est aussi affirmer une éthique de l'écriture qui ne s'affiche plus dans le circuit commercial de l'inédit mais travaille en profondeur les universaux littéraires. Il va de soi alors que la perspective «nationaliste» de l'expression romanesque devienne secondaire.

\section{Bibliographie}

Aubague, Laurent, Franco, Jean et Lara-Alengrin, Alba (dir.), 2009, Les littératures d'Amérique latine au $X X^{e}$ siècle: une poétique de la transgression?, Paris, L'Harmattan.

Berchtold, Jacques (dir.), 1998, Echiquiers d'encre. Le Jeu d'échecs et les Lettres (XIX ${ }^{e}$-XX ${ }^{e}$ s.), Genève, Droz.

Blanchot, Maurice, 1969, L'entretien infini, Paris, Gallimard, NRF. 
Borges, Jorge Luis, 2009, Poesía completa, Barcelona, Ediciones Destino, Colección Áncora y Delfín, Volumen 1093.

Borges, Jorge Luis, 1970, Oeuvre poétique 1925-1965, Paris, Gallimard, NRF.

Carlos González Boixo (ed.), José, 2009, Tendencias de la narrativa mexicana actual, Madrid, Iberoamericana, Francfort, Vervuert Verlag, Mexico, Bonilla Artigas Editores.

Dostoïevski, Fiodor, 1999, Le Double, Arles, Actes Sud, Babel.

Padilla, Ignacio, 2000, Amphitryon, Madrid, Editorial Espasa Calpe.

Pessoa, Fernando, 1987, Poesia I. 1902-1929, Lisboa, Publicacões Europa-América, collection Livros de bolso Europa-América, $n^{\circ} 436$.

Pessoa, Fernando, 2001, Oeuvres poétiques, Paris, Gallimard, NRF, Bibliothèque de la Pléiade.

Pluta, Nina, 2009, «El género seudocriminal. Inspiraciones policíacas en las novelas mexicanas del cambio de siglo» in Carlos González Boixo (ed.), José, Tendencias de la narrativa mexicana actual, Madrid, Iberoamericana, Francfort, Vervuert Verlag, Mexico, Bonilla Artigas Editores, p. 201-228.

Volpi, Jorge, Urroz, Eloy, Padilla, Ignacio, Chávez, Palou, Miguel Ángel, 1996, Manifiesto Crack: http://www.lai.at/wissenschaft/lehrgang/semester/ss2005/fs/ files/crack.pdf 